\title{
Synchronization-free all-solid-state laser system for stimulated Raman scattering microscopy
}

\author{
Tobias Steinle ${ }^{1}$, Vikas Kumar ${ }^{2}$, Moritz Floess ${ }^{1}$, Andy Steinmann ${ }^{1}$, Marco Marangoni², Claudia Koch ${ }^{3}$, \\ Christina Wege ${ }^{3}$, Giulio Cerullo ${ }^{2}$ and Harald Giessen ${ }^{1}$
}

We introduce an extremely simple and highly stable system for stimulated Raman scattering (SRS) microscopy. An 8-W, 450-fs Yb:KGW bulk oscillator with $41 \mathrm{MHz}$ repetition rate pumps an optical parametric amplifier, which is seeded by a cw tunable external cavity diode laser. The output radiation is frequency doubled in a long PPLN crystal and generates 1.5-ps long narrowband pump pulses that are tunable between 760 and $820 \mathrm{~nm}$ with $>50 \mathrm{~mW}$ average power. Part of the oscillator output is sent through an etalon and creates Stokes pulses with $100 \mathrm{~mW}$ average power and $1.7 \mathrm{ps}$ duration. We demonstrate SRS microscopy at a $30-\mu \mathrm{s}$ pixel dwell time with high chemical contrast, signal-to-noise ratio in excess of 45 and no need for balanced detection, thanks to the favorable noise properties of the bulk solid-state system. $\mathrm{Cw}$ seeding intrinsically ensures low spectral drift. We discuss its application to chemical contrast microscopy of freshly prepared plant tissue sections at different vibrational bands.

Light: Science \& Applications (2016) 5, e16149; doi:10.1038/lsa.2016.149; published online 7 October 2016

Keywords: label-free imaging; stimulated Raman scattering; optical parametric amplifier; spectral compression

\section{INTRODUCTION}

Coherent Raman scattering (CRS) is a powerful nonlinear microscopy technique for label-free, three-dimensional imaging of tissues and cells, exploiting the characteristic vibrational response of molecules as an intrinsic contrast mechanism ${ }^{1}$. CRS enables real-time, nondestructive chemical identification of compounds at the submicrometer scale without addition of any external marker ${ }^{2}$, whose presence could perturb the molecules under study or even inhibit the evolution of dynamical processes, such as drug transport or cell differentiation. CRS microscopy has found many applications in biomedical optics ${ }^{3,4}$; in particular, given the strong Raman response of long-chain hydrocarbons, it is the ideal method for visualizing lipids and studying their metabolism ${ }^{5}$, as well as for differentiating lipids, DNA, and proteins within the cell ${ }^{6}$. In addition CRS promises to be able to rapidly discriminate between tumors and healthy tissue, paving the way to 'virtual histopathology', both ex vivo and intraoperatively ${ }^{7,8}$.

CRS techniques require two synchronized laser pulses at frequencies $\omega_{\mathrm{p}}$ (pump frequency) and $\omega_{\mathrm{S}}$ (Stokes frequency) to drive a vibrational coherence within the ensemble of molecules inside the laser focus. When the difference between pump and Stokes frequencies matches a specific vibrational frequency $\Omega$, i.e., $\omega_{\mathrm{p}}-\omega_{\mathrm{S}}=\Omega$, then all the molecules in the focal volume vibrate in phase, enhancing the Raman response by many orders of magnitude with respect to the incoherent, spontaneous Raman process. The two most widely employed CRS techniques are coherent anti-Stokes Raman scattering (CARS) ${ }^{9}$ and stimulated Raman scattering (SRS $)^{10,11}$. In the CARS process the vibrational coherence is read by a further interaction with the pump beam, generating radiation at the anti-Stokes frequency $\omega_{\mathrm{aS}}=\omega_{\mathrm{p}}+\Omega$; in SRS coherent interaction with the sample induces stimulated emission from a virtual state of the sample to the investigated vibrational state, resulting in a Stokes-field amplification (stimulated Raman gain, SRG) and in a simultaneous pump-field attenuation (stimulated Raman loss, SRL). CARS can be more simply implemented, since the nonlinear signal is at a frequency $\omega_{\mathrm{a} S}$, differing from those of pump and Stokes, and can thus be easily singled out by spectral filtering. SRS is instrumentally more demanding, since it requires the measurement of a tiny differential signal (the SRG or SRL) on top of a large and potentially noisy background (the Stokes or pump intensity). On the other hand, SRS has two important advantages: (i) it is free from non-resonant background, generated by four-wave-mixing ${ }^{12}$, which in CARS adds to and often obscures the resonant signal of interest; (ii) it provides a signal that is linear with the density of oscillators, thus allowing quantitative evaluation of the concentrations of the molecular species ${ }^{13}$. In broadband or spectroscopic CARS the non-resonant background acts as a phase-locked local oscillator heterodyning the resonant signal, which can be recovered by numerical processing ${ }^{14}$; this procedure is, however, not possible in the single-frequency mode, which allows the highest acquisition speeds up to the video rate $e^{15,16}$. For these reasons SRS is currently the technique of choice for a broad spectrum of applications.

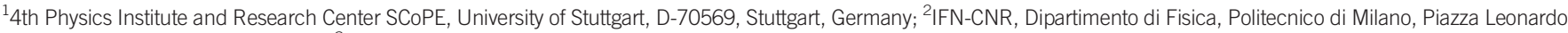
da Vinci 32, I-20133, Milan, Italy and ${ }^{3}$ Institute of Biomaterials and Biomolecular Systems, Dpt. of Molecular Biology and Plant Virology, University of Stuttgart, Pfaffenwaldring 57 , D-70569, Stuttgart, Germany

Correspondence: T Steinle, Email: t.steinle@pi4.uni-stuttgart.de

Received 22 December 2015; revised 1 April 2016; accepted 5 April 2016; accepted article preview online 7 April 2016 
The main stumbling block which has so far prevented widespread adoption of CRS microscopy and, in particular, SRS in the biological and medical communities is the complication and cost of the experimental apparatus. Raman transitions exhibit linewidths of the order of tens of $\mathrm{cm}^{-1}$ in the condensed phase: To efficiently excite them with the highest peak power, while still preserving frequency resolution and molecular selectivity, picosecond pump/Stokes pulses are thus needed. In its single-frequency version, CRS microscopy requires two synchronized trains of narrowband, picosecond pulses (pump and Stokes) with tunable frequency difference, high repetition rate $(\approx 100 \mathrm{MHz})$ and output power $\approx 100 \mathrm{~mW}$ per branch, which is required to compensate for losses in the optical chain of the microscope. The complexity of the excitation laser is one reason why, after its early demonstration in $1982^{17}$, the development of CRS microscopy has stopped for nearly two decades. Following its revival, initial CRS implementations were based on two electronically synchronized picosecond Ti:sapphire oscillators ${ }^{18}$, while the current 'gold standard' is represented by an optical parametric oscillator (OPO) synchronously pumped by a picosecond $\mathrm{Nd} \mathrm{YVO}_{4}$ oscillator ${ }^{19}$. Such systems are complex, expensive, and they all critically require the synchronization between two independent laser sources, which must be maintained over time. Drastically simplified excitation architectures, with lower cost and smaller footprint, are thus greatly in demand, as they would lower the technological entrance barriers to CRS microscopy.

In recent years, considerable efforts have been devoted to the development of fiber-format systems for CRS microscopy, as they are intrinsically compact and turn-key. Several architectures, based on Eror Yb-doped fibers combined with nonlinear spectral broadening and frequency conversion, have been demonstrated ${ }^{20-29}$. While these systems have been successfully applied to CARS imaging ${ }^{26-29}$, the SRS modality has been much harder and in some cases impossible ${ }^{28}$ to be implemented. This is due to the fact that fiber lasers intrinsically suffer from excess high-frequency noise with respect to their bulk counterparts, because of the much greater length of the active medium which leads to higher amplified spontaneous emission levels. This extra noise pedestal, which can amount to as much as $30 \mathrm{~dB}$, prevents sensitive detection of the weak nonlinear SRS signal, sitting on top of the strong and fluctuating Stokes (pump) signal, unless balanced detection schemes ${ }^{22,23,30}$ with sophisticated noise-cancelling electronics $^{23}$ or complex optical layouts ${ }^{30}$ are used. Such schemes become particularly challenging to be implemented when measuring biological samples, with large variations of transmission/scattering over the scanned area.

Recently we introduced a novel compact and low-cost architecture for CRS microscopy based on a bulk all-solid-state Yb:KGW oscillator followed by an optical parametric amplifier (OPA), employing periodically poled lithium niobate $(\mathrm{PPLN})^{31}$. OPAs are significantly simpler than OPOs, as they work without a feedback cavity. However, as they require high nonlinear gain levels they are typically driven by amplified laser systems. In our case the high peak power of the $\mathrm{Yb}$ oscillator, combined with the large nonlinear coefficient of PPLN, allowed its use to directly drive the OPA at tens of $\mathrm{MHz}$ repetition rate, while preserving the excellent noise figure of the bulk solid-state laser. However, a critical aspect of an OPA is the generation of the seed light, which typically requires a nonlinear optical process starting from the pump. Our previous implementation still used a complex optical layout with several OPA stages, and self-phase-modulation in a tapered fiber was required to generate a tunable OPA seed. Here we demonstrate a drastic simplification of the system, in which the OPA is seeded by a tunable continuous wave external-cavity diode laser (ECDL). This configuration completely removes path-length synchronization issues, greatly simplifying the optical setup and enhancing its robustness and insensitivity to environmental perturbations. Exploiting the very low noise of solid-state bulk oscillators in the $\mathrm{MHz}$ regime, we demonstrate high speed, low-noise SRS imaging without balanced detection.

\section{MATERIALS AND METHODS}

Our SRS system as shown in Figure 1 is based on a home-built modelocked Yb:KGW solid-state oscillator, which provides up to $8 \mathrm{~W}$ of output power (with $6.2 \mathrm{~W}$ available for the experiment) at $1032 \mathrm{~nm}$ with 450 -fs pulse width at a repetition rate of $41 \mathrm{MHz}^{32}$. A $1.5-\mathrm{W}$ fraction of the main laser output is used to pump a single-stage OPA based on a 10-mm-long MgO-doped PPLN crystal, equipped with 9 different poling periods (from $\Lambda=27.91 \mu \mathrm{m}$ to $\Lambda=31.59 \mu \mathrm{m}$ ). The OPA is seeded by a home-built cw ECDL (gain chip: SAL:1590-100, Sacher Lasertechnik), employing a grating in the Littrow configuration, and generating $30 \mathrm{~mW}$ average power over the $1500-1650 \mathrm{~nm}$ tuning range with single longitudinal mode operation. Its fibered output provides after collimation a nearly diffraction-limited beam that is mode-matched and collinearly combined with the $\mathrm{Yb}: \mathrm{KGW}$ output and focused to a 50- $\mu \mathrm{m}$ waist in the PPLN crystal. Cw seeding dramatically simplifies the OPA operation, as there is no need for

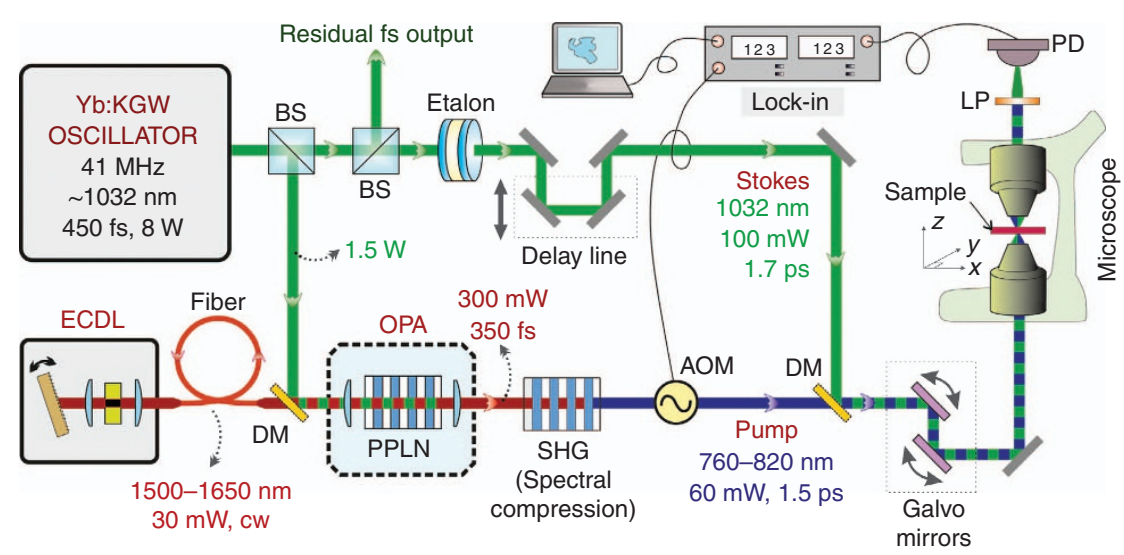

Figure 1 Schematic diagram of the experimental system used for stimulated Raman scattering microscopy. AOM, acousto-optic modulator; BS, beam splitter; DM, dichroic mirror; ECDL, external-cavity diode laser; LP, long pass filter; PD, amplified silicon photo diode; PPLN, periodically-poled lithium niobate. 
pump-seed synchronization ${ }^{33}$. Considering the ECDL output power, the pump pulse duration and the repetition rate, there are approximately $10^{5}$ seed photons per pulse; this level is sufficient to completely suppress parametric superfluorescence in the OPA, greatly improving the energy stability, removing timing jitter ${ }^{34}$, and minimizing spectral drift. Further, it enables precise adjustment of the OPA center wavelength by rotating the ECDL grating and changing poling period and crystal temperature accordingly. In the future, a fan-out design will allow for simpler, faster and gapless spectral tuning. Ultimately, using commercially available ECDLs and fast linear translation stages in combination with fan-out crystals, a tuning speed over the entire $\mathrm{CH}$-region below $1 \mathrm{~s}$ can be realized. Thanks to the high nonlinear coefficient of PPLN, the output power remains above the $300 \mathrm{~mW}$ level across the whole tuning range of the ECDL (Figure 2, lower panel). The spectral bandwidth amounts to $10 \mathrm{~nm}$, dictated by the linewidth of the $\mathrm{Yb}$ oscillator, which corresponds to a pulse duration of $\approx 350$ fs. The Raman pump pulses for the SRS process are obtained by frequency doubling the OPA output in a second 10-mm-long PPLN crystal and exploiting second-harmonic generation (SHG) spectral compression ${ }^{35}$ to reduce the bandwidth to $\approx 11 \mathrm{~cm}^{-1}$. A tuning range between 760 and $820 \mathrm{~nm}$, as limited by the available poling periods of the SHG crystal, is obtained with power levels $>50 \mathrm{~mW}$ (Figure 2, upper panel). The corresponding pumpStokes frequency detuning ranges from 2500 to $3460 \mathrm{~cm}^{-1}$, thus fully covering the $\mathrm{C}-\mathrm{H}$ stretching band that is typically used for the imaging of lipids. By replacing the ECDL and the PPLN crystals, the OPA tuning range can be easily extended to $1770-1870 \mathrm{~nm}$, thus allowing the $1000-1600 \mathrm{~cm}^{-1}$ fingerprint region to be covered after SHG. The fixed frequency 1032-nm Stokes pulses are created from the residual oscillator power by spectral filtering with an etalon with a free spectral range of $85 \mathrm{~cm}^{-1}$ and a finesse of 12 , resulting in pulses with $\approx 8 \mathrm{~cm}^{-1}$ bandwidth. Considering the $28 \%$ transmission of the etalon, the $4.5 \mathrm{~W}$ of residual oscillator power would result in $1.26 \mathrm{~W}$ maximum Stokes power. However, since typically $<100 \mathrm{~mW}$ will be needed, the
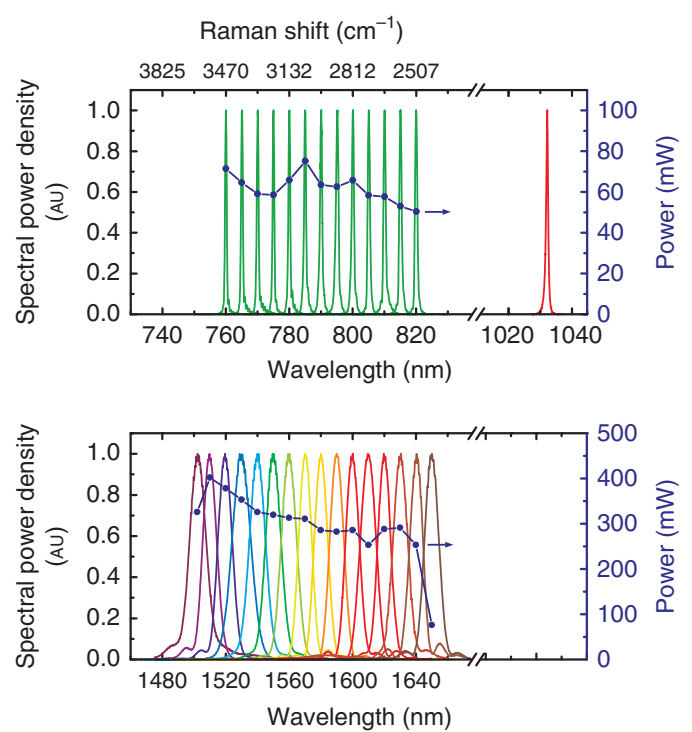

Figure 2 Power-spectra of external-cavity diode laser seeded OPA (lower panel). Corresponding SHG from PPLN (in green in upper panel) serving as tunable Raman pump. The upper panel also depicts the spectrum of the Stokes beam, filtered by an etalon. remaining oscillator power is available for other nonlinear optical microscopy modalities or even to pump additional OPAs.

Pump and Stokes pulses are synchronized by a delay line, collinearly combined by a dichroic beam-splitter and sent to a home-built laserscanning SRS microscope, working in transmission mode and using a $\mathrm{NA}=0.7$ focusing objective (Nikon 60x). The transmitted light is collected with a NA $=1.4$ achromatic condenser. We chose to detect the SRG of the Stokes beam, which benefits from the extremely good noise properties of the all-solid-state Yb:KGW oscillator. An acoustooptic modulator is used to modulate the pump pulses at $500 \mathrm{kHz}$, while the Stokes intensity is detected by a single-channel amplified silicon photodiode, which is connected to a high-frequency lock-in amplifier. A pair of galvanometric mirrors is used for the fast spatial scanning of the samples.

\section{RESULTS AND DISCUSSION}

We first validated the system by measuring SRS spectra of various solvents. Figure 3 shows the SRG spectrum of an acetone solution, as measured by scanning the pump wavelength from 777 to $802 \mathrm{~nm}$ : the spectrum and in particular the position of the characteristic peak at $2923 \mathrm{~cm}^{-1}$ is in very good agreement with the spontaneous Raman spectrum, displayed in the inset of Figure 3a, as expected for SRS, which directly measures the imaginary part of the third-order nonlinear susceptibility without lineshape distortions ${ }^{10,11}$. The stability of the SRS source is monitored in a continuous 24 -h long time measurement of the SRG signal at the acetone peak at $2923 \mathrm{~cm}^{-1}$. The system was free-running without any active control or feedback during the whole time. The average SRG signal drift is lower than 3\% over this period, with a peak-to-peak difference of $13 \%$, as shown in Figure 3b. Note that the SRG signal depends on multiple parameters, such as pump and Stokes power, wavelength detuning and beam pointing fluctuation; its very low long-term drift without active stabilization is a clear indication of the intrinsic stability of our design. Due to the absence of synchronization issues, the system can provide this performance even after a cold start of the $\mathrm{Yb}$ oscillator and the ECDL.

a
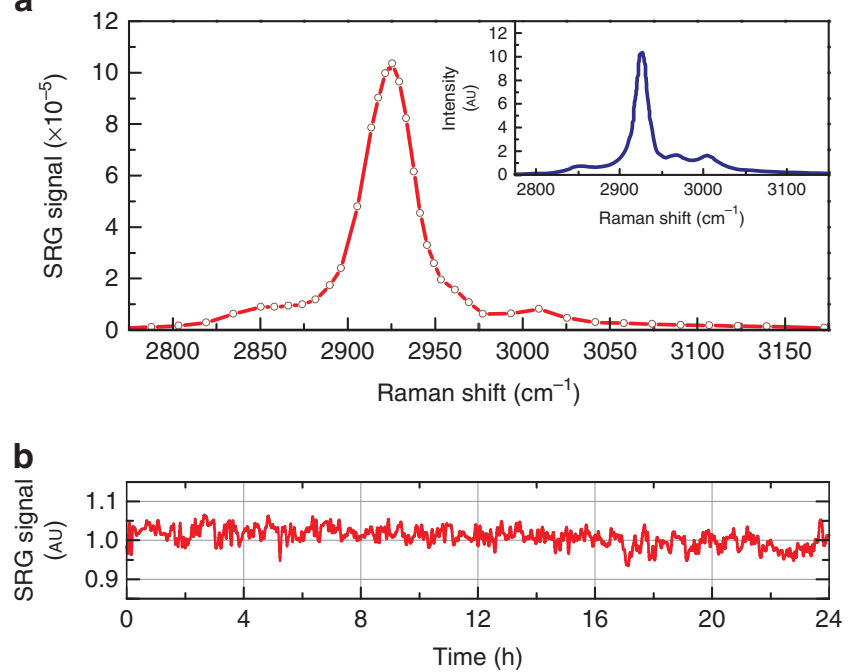

Figure 3 (a) SRG spectra of acetone solution compared to the spontaneous Raman spectrum shown in inset. (b) Stability of the SRG signal over $24 \mathrm{~h}$ at the acetone resonance of $2923 \mathrm{~cm}^{-1}$. The signal is a 5 -minute running average, sampled with $1 \mathrm{~Hz}$. 

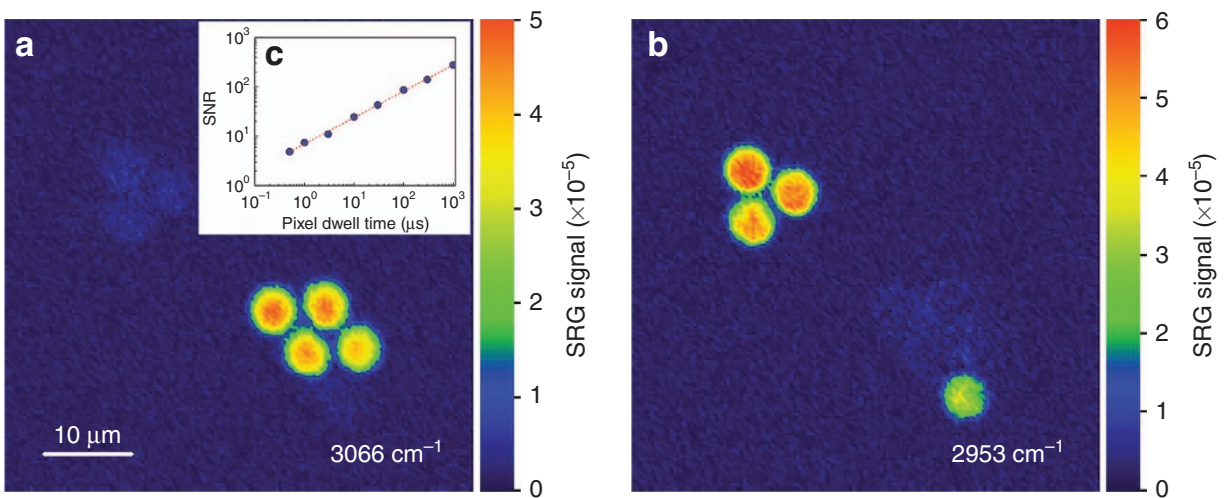

Figure 4 (a) and (b): Stimulated Raman gain images $(54 \times 54 \mu \mathrm{m}, 150 \times 150$ pixels) of a blend of 6 - $\mu \mathrm{m}$ PS and PMMA beads taken at their respective Raman resonances of 3066 and $2953 \mathrm{~cm}^{-1}$. These images are acquired with $30 \mu \mathrm{s}$ pixel dwell time with $16 \mathrm{~mW}$ pump (modulated at $500 \mathrm{kHz}$ ) and $2 \mathrm{~mW}$ Stokes powers respectively, at the sample. The focusing microscope objective (Nikon 60x, 0.70 NA in focus) has $~ 79 \%$ and $44 \%$ transparencies for pump and the Stokes wavelengths, respectively. The inset graph (c) shows the measured dependence of the signal-to-noise ratio on integration time for acquisition of PS beads under the same experimental conditions.

a

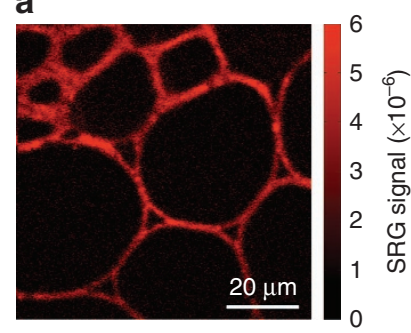

d

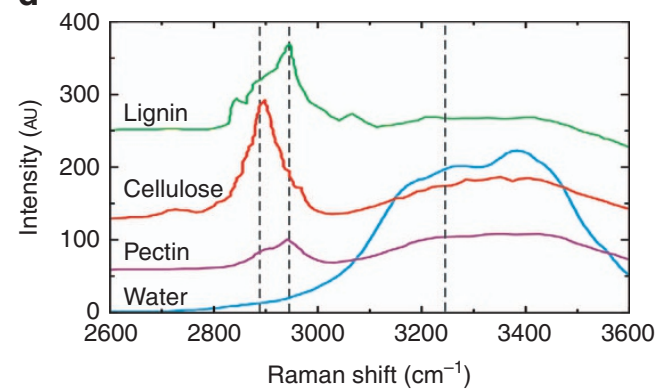

b

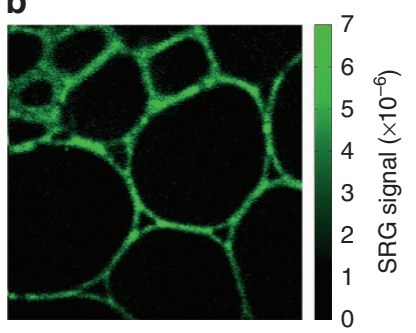

C

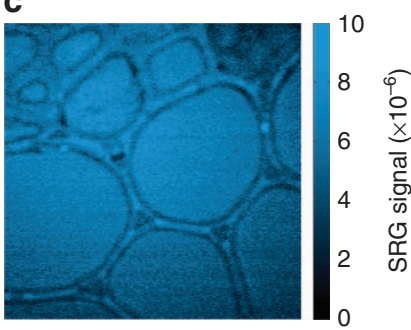

e

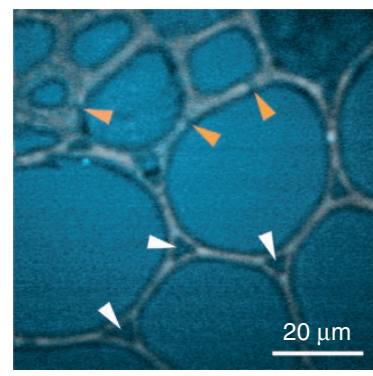

Figure 5 (a-c) SRG images $(80 \times 80 \mu \mathrm{m}, 400 \times 400$ pixels) of cells in an cross-sectional area of Abutilon pictum leaf petiole, containing sclerenchyma (upper left area) and parenchyma cells, acquired at $300 \mu$ s integration time at pump-Stokes frequency detunings of 2890,2945 , and $3245 \mathrm{~cm}^{-1}$, respectively. (d) Raman spectra, available in the literature, of key compounds present in the plant cells: polysaccharides including cellulose and pectin, polyphenols (lignin), and water. The intensity in the $2800-3000 \mathrm{~cm}^{-1}$ region of the Raman spectrum originates mainly from $\mathrm{C}-\mathrm{H}$ stretching vibrations, while the intensity in the $3050-3600 \mathrm{~cm}^{-1}$ region mainly stems from $\mathrm{O}-\mathrm{H}$ stretching vibrations. (e) Overlay image of all three SRG signals. Exemplary sites of intercellular spaces are indicated by white arrowheads, pits, or plasmodesmata by orange arrowheads. Refer to text for details.

As a first test of the imaging capabilities of our SRS setup, we used a composite sample of poly-methyl methacrylate (PMMA) and polystyrene (PS) beads with a $6-\mu \mathrm{m}$ diameter, and probed it at frequency detunings of 2953 and $3066 \mathrm{~cm}^{-1}$. Figure 4a shows the image at $3066 \mathrm{~cm}^{-1}$, corresponding to the resonance of the PS beads. Chemical contrast is demonstrated by tuning to $2953 \mathrm{~cm}^{-1}$, corresponding to the resonance of the PMMA beads. In both cases the average power levels are $16 \mathrm{~mW}$ (pump) and $2 \mathrm{~mW}$ (Stokes) on the sample. The pixel dwell time is $30 \mu$ s, so that the acquisition of a full image $(150 \times 150$ pixels $)$ takes less than one second. Despite the relatively low value of SRG signal $\left(<6 \times 10^{-5}\right)$ the images are very clean, with a signal-to-noise ratio ( $\mathrm{SNR}$ ) as high as 45 without the use of balanced detection. We acquired images with different speeds; the inset of Figure $4 \mathrm{a}$ shows the SNR of the image as a function of pixel dwell time, which displays the expected square root scaling. A comparison of the imaging capabilities of our ECDL-OPA system to an OPO system is given in the Supplementary Material. This level of performance favorably compares with that reported so far for green-pumped picosecond OPOs, while surpassing those obtained with fiber lasers, which suffer from high intensity noise in the radiofrequency domain, preventing SRS imaging with direct detection. 
To demonstrate the performance of the system on a biological sample, we imaged a tissue domain of a leaf petiole prepared from an Abutilon pictum plant. Figure 5 shows SRG images of an $80 \times 80 \mu \mathrm{m}$ cross-sectional area of this stem, containing thick-walled sclerenchyma cells (small lumina; top left) and large parenchyma cells (right), at three different pump-Stokes frequency detunings, namely $2890 \mathrm{~cm}^{-1}$ (Figure 5a), $2945 \mathrm{~cm}^{-1}$ (Figure 5b), and $3245 \mathrm{~cm}^{-1}$ (Figure 5c). In Figure $5 \mathrm{a}$ and $\mathrm{b}$, the plant cell walls are clearly visible due to the SRG signal originating from the $\mathrm{C}-\mathrm{H}$ stretching vibrations of the molecules of their major constituents, namely the macromolecular polysaccharides cellulose, xyloglucans, and pectins, as well as xylans and polyphenolic polymers (lignins) ${ }^{36}$, whose Raman resonances (see spontaneous Raman spectra in Figure 5d) correspond to the adopted frequency detunings. On the other hand, in Figure $5 c$ the SRG signal is dominated by the $\mathrm{O}-\mathrm{H}$ stretching vibrations of the water molecules, which are homogeneously distributed within the lumina of the cells. In-between adjacent cells, intercellular spaces formed by separation of contiguous walls at junctions of three or more cells can be seen (as indicated exemplarily by white arrowheads in 5e). Pit canals traversing the cell walls, with plasmodesmata as connections between the living protoplasts of adjoining cells, are discernible (exemplified by orange arrowheads).The overlay image $5 \mathrm{e}$ in a pseudo-color scheme, using same color assignments for identification, illustrates the combined distribution of contents and their appearances over the scanned specimen area.

\section{CONCLUSIONS}

In this paper we present a novel compact, all-solid-state system for SRS microscopy, based on a mode-locked bulk Yb:KGW oscillator driving an ECDL-seeded OPA. This is a passive, single-pass system, which avoids third-order nonlinearities and synchronization issues, since no delay control is required for the tunable Raman pump generation. Hence, it is not only simpler to set up and operate, with respect to existing solutions based on bulk lasers, but also robust with respect to thermal or environmental changes and well suitable for long term operation. At the same time, our approach avoids the excess high-frequency noise intrinsic in fiber-based laser systems, allowing high-quality SRS imaging without complex balanced detection schemes. In addition, with respect to other solutions for SRS microscopy, our system offers the following important advantages:

i The bandwidths of both pump and Stokes pulses can be easily adjusted to match the linewidth of the vibration of interest by selecting the etalon design and the length of the SHG crystal used for spectral compression ${ }^{35}$; we chose bandwidths of $10-20 \mathrm{~cm}^{-1}$, corresponding to a pulse duration of $1-2 \mathrm{ps}$, which is the optimum compromise between peak power and spectral resolution for lipid imaging ${ }^{37}$.

ii The system also produces high peak power 450 -fs pulses, which can be spectrally broadened in a nonlinear fiber and compressed to sub-100-fs duration; these pulses are available for other nonlinear microscopy modalities, such as second and third harmonic imaging or two-photon excited fluorescence.

We believe that our system will significantly reduce the technical entrance barriers to SRS microscopy for label-free chemical contrast imaging, bringing it closer to real-world applications in biomedical and biological research and in diagnostics.

\section{CONFLICT OF INTEREST}

The authors declare no conflict of interest.

\section{ACKNOWLEDGEMENTS}

We thank Sigrid Kober for many helpful discussions and the supply of living plant samples. We acknowledge support by the Carl Zeiss foundation and the Projekthaus NanoBioMater at the University of Stuttgart, ERC (ComplexPlas), EU-COST (MP1302), the EU Graphene Flagship (contract no. CNECT-ICT604391), BW-Stiftung, Deutsche Forschungsgemeinschaft, BMBF, as well as by Fondazione Cariplo through the 2012-0904 project.

1 Min W, Freudiger CW, Lu SJ, Xie XS. Coherent nonlinear optical imaging: beyond fluorescence microscopy. Annu Rev Phys Chem 2011; 62: 507-530.

2 Camp CH, Cicerone MT. Chemically sensitive bioimaging with coherent Raman scattering. Nat Photon 2015; 9: 295-305.

3 Pezacki JP, Blake JA, Danielson DC, Kennedy DC, Lyn RK et al. Chemical contrast for imaging living systems: molecular vibrations drive CARS microscopy. Nat Chem Biol 2011; 7: 137-145.

4 Yu Y, Ramachandran PV, Wang MC. Shedding new light on lipid functions with CARS and SRS microscopy. Biochim Biophys Acta 2014; 1841: 1120-1129.

5 Zumbusch A, Langbein W, Borri P. Nonlinear vibrational microscopy applied to lipid biology. Progress Lipid Res 2013; 52: 615-632.

6 Lu FK, Basu S, Igras V, Hoang MP, Ji MB et al. Label-free DNA imaging in vivo with stimulated Raman scattering microscopy. Proc Natl Acad Sci USA 2015; 112 : $11624-11629$.

7 Ji MB, Orringer DA, Freudiger CW, Ramkissoon S, Liu XH et al. Rapid, label-free detection of brain tumors with stimulated Raman scattering microscopy. Sci Trans/ Med 2013; 5: 201ra119.

$8 \mathrm{Ji}$ MB, Lewis S, Camelo-Piragua S, Ramkissoon SH, Snuderl M et al. Detection of human brain tumor infiltration with quantitative stimulated Raman scattering microscopy. Sci Transl Med 2015; 7: 309ra163.

9 Zumbusch A, Holtom GR, Xie XS. Three-dimensional vibrational imaging by coherent anti-Stokes Raman scattering. Phys Rev Lett 1999; 82: 4142-4145.

10 Freudiger CW, Min W, Saar BG, Lu SJ, Holtom GR et al. Label-free biomedical imaging with high sensitivity by stimulated Raman scattering microscopy. Science 2008; 322 : 1857-1861.

11 Nandakumar P, Kovalev A, Volkmer A. Vibrational imaging based on stimulated Raman scattering microscopy. New J Phys 2009; 11: 033026.

12 Evans CL, Xie XS. Coherent anti-Stokes Raman scattering microscopy: chemical imaging for biology and medicine. Annu Rev Anal Chem 2008; 1: 883-909.

13 Fu D, Zhou J, Zhu WS, Manley PW, Wang YK et al. Imaging the intracellular distribution of tyrosine kinase inhibitors in living cells with quantitative hyperspectral stimulated Raman scattering. Nat Chem 2014; 6: 614-622.

14 Camp CH Jr, Lee YJ, Heddleston JM, Hartshorn CM, Walker ARH et al. High-speed coherent Raman fingerprint imaging of biological tissues. Nat Photon 2014; 8: $627-634$.

15 Evans CL, Potma EO, Puoris'haag M, Cote D, Lin CP et al. Chemical imaging of tissue in vivo with video-rate coherent anti-Stokes Raman scattering microscopy. Proc Nat Acad Sci USA 2005; 102: 16807-16812.

16 Saar BG, Freudiger CW, Reichman J, Stanley CM, Holtom GR et al. Video-rate molecular imaging in vivo with stimulated Raman scattering. Science 2010; 330: 1368-1370.

17 Duncan MD, Reintjes J, Manuccia TJ. Scanning coherent anti-Stokes Raman microscope. Opt Lett 1982; 7: 350-352.

18 Potma EO, Jones DJ, Cheng JX, Xie XS, Ye J. High-sensitivity coherent anti-Stokes Raman scattering microscopy with two tightly synchronized picosecond lasers. Opt Let 2002; 27: 1168-1170.

19 Ganikhanov F, Carrasco S, Xie XS, Katz M, Seitz W et al. Broadly tunable dual-wavelength light source for coherent anti-Stokes Raman scattering microscopy. Opt Lett 2006; 31: 1292-1294.

20 Krauss G, Hanke T, Sell A, Träutlein D, Leitenstorfer A et al. Compact coherent anti-Stokes Raman scattering microscope based on a picosecond two-color Er: fiber laser system. Opt Lett 2009; 34: 2847-2849.

21 Marangoni M, Gambetta A, Manzoni C, Kumar V, Ramponi R et al. Fiber-format CARS spectroscopy by spectral compression of femtosecond pulses from a single laser oscillator. Opt Lett 2009; 34: 3262-3264.

22 Gambetta A, Kumar V, Grancini G, Polli D, Ramponi R et al. Fiber-format stimulatedRaman-scattering microscopy from a single laser oscillator. Opt Lett 2010; 35 : 226-228.

23 Freudiger CW, Yang WL, Holtom GR, Peyghambarian N, Xie XS et al. Stimulated Raman scattering microscopy with a robust fibre laser source. Nat Photon 2014; 8: 153-159.

24 Coluccelli N, Kumar V, Cassinerio M, Galzerano G, Marangoni M et al. Er/Tm: fiber laser system for coherent Raman microscopy. Opt Lett 2014; 39: 3090-3093.

25 Baumgartl M, Chemnitz M, Jauregui C, Meyer T, Dietzek B et al. All-fiber laser source for CARS microscopy based on fiber optical parametric frequency conversion. Opt Express 2012; 20: 4484-4493. 
26 Gottschall T, Baumgartl M, Sagnier A, Rothhardt J, Jauregui C et al. Fiber-based source for multiplex-CARS microscopy based on degenerate four-wave mixing. Opt Express 2012; 20: 12004-12013.

27 Chemnitz M, Baumgartl M, Meyer T, Jauregui C, Dietzek B et al. Widely tuneable fiber optical parametric amplifier for coherent anti-Stokes Raman scattering microscopy. Opt Express 2012; 20: 26583-26595.

28 Lefrancois S, Fu D, Holtom GR, Kong LJ, Wadsworth WJ et al. Fiber four-wave mixing source for coherent anti-Stokes Raman scattering microscopy. Opt Lett 2012; 37: 1652-1654.

29 Lamb ES, Lefrancois S, Ji MB, Wadsworth WJ, Xie XS et al. Fiber optical parametric oscillator for coherent anti-Stokes Raman scattering microscopy. Opt Lett 2013; 38: 4154-4157.

30 Nose K, Ozeki Y, Kishi T, Sumimura K, Nishizawa N et al. Sensitivity enhancement of fiber-laser-based stimulated Raman scattering microscopy by collinear balanced detection technique. Opt Express 2012; 20: 13958-13965.

31 Steinle T, Kumar V, Steinmann A, Marangoni M, Cerullo G et al. Compact, low-noise, all-solid-state laser system for stimulated Raman scattering microscopy. Opt Lett 2015; 40: 593-596.

32 Steinmann A, Metzger B, Hegenbarth R, Giessen H. Compact 7.4W femtosecond oscillator for white-light generation and nonlinear microscopy. Proceedings of the Conference on Lasers and Electro-Optics 2011; paper CThAA5. Baltimore, Maryland, USA: CLEO, 2011.

33 Steinle T, Kedenburg S, Steinmann A, Giessen $\mathrm{H}$. Combining $\mathrm{cW}$-seeding with highly nonlinear fibers in a broadly tunable femtosecond optical parametric amplifier at 42 $\mathrm{MHz}$. Opt Lett 2014; 39: 4851-4854.
34 Manzoni C, Cirmi G, Brida D, De Silvestri S, Cerullo G. Optical-parametric-generation process driven by femtosecond pulses: Timing and carrier-envelope phase properties. Phys Rev A 2009; 79: 033818.

35 Marangoni M, Brida D, Quintavalle M, Cirmi G, Pigozzo FM et al. Narrow-bandwidth picosecond pulses by spectral compression of femtosecond pulses in a second-order nonlinear crystal. Opt Express 2007; 15: 8884-8891.

36 Evert RF. Esau's Plant Anatomy: Meristems, Cells, and Tissues of the Plant Body - Their Structure, Function, and Development. Hoboken, New Jersey: John Wiley \& Sons, Inc., 3rd ed, 2006.

37 Rimke I, Hehl G, Beutler M, Volz P, Volkmer A et al. Tunable dual-wavelength two-picosecond light source for coherent Raman scattering microscopy. In: Periasamy A, So PTC, König Keditors. Proceedings of SPIE Multiphoton Microscopy in the Biomedical Sciences XIV. San Francisco, CA, USA: SPIE, 2014; 8948: 894816.

(c) (i) $\ominus$ This work is licensed under a Creative Commons AttributionCy NC ND NonCommercial-NoDerivs 4.0 International License. The images or other third party material in this article are included in the article's Creative Commons license, unless indicated otherwise in the credit line; if the material is not included under the Creative Commons license, users will need to obtain permission from the license holder to reproduce the material. To view a copy of this license, visit http:// creativecommons.org/licenses/by-nc-nd/4.0/

(C) The Author(s) 2016

Supplementary Information for this article can be found on the Light: Science \& Applications' website (http://www.nature.com/lsa). 